\title{
Effet des enrichissements en nutriments sur le phytoplancton isolé à l'aide d'enceintes expérimentales placées in situ dans deux lacs réservoirs de la région de Marrakech (Maroc)
}

\author{
K. Mouhri ${ }^{1}$ \\ M. Loudiki ${ }^{1}$ \\ R. Le Cohu ${ }^{2}$ \\ N. Zaki1
}

Mots-clés : Lac-réservoir, phytoplancton, enceintes, enrichissement, nutriments, croissance.

Ce travail a pour objectif d'évaluer l'effet d'apports en nutriments (en particulier azote et phosphore) sur la croissance et la composition du phytoplancton dans deux lacs-réservoirs de la région de Marrakech (Hassan 1 et Lalla Takerkoust).

L'approche expérimentale est basée sur l'incubation du phytoplancton à l'intérieur d'enceintes imperméables en polyéthylène enrichies en azote ou phosphore ou les deux en combinaison. Le temps d'incubation varie de 8 à 15 jours. Les résultats des différentes expériences d'enrichissement ont permis de dégager les points suivants :

- l'ajout de l'azote seul ne présente aucun effet significatif sur la croissance du phytoplancton ;

- la fertilisation par le phosphore seul ou en combinaison avec l'azote entraîne un fort accroissement du peuplement phytoplanctonique. Le phosphore semble donc constituer le premier facteur limitant le développement du phytoplancton dans les deux lacs étudiés bien que leur concentration moyenne en phosphore et leur degré de trophie soient complètement différents. L'azote pour sa part semble constituer le second facteur limitant la production primaire dans les deux lacs-réservoirs.

Effect of nutrient enrichments on phytoplankton isolated by in situ experimental enclosures placed in two lakes of the Marrakech area (Morocco)

Keywords : Reservoir lake, phytoplankton, enclosures, nutrients, enrichment, growth.

The aim of the present work is to evaluate the effect of nutrient supplies, particularly nitrogen and phosphorus, on phytoplankton growth and composition in two lakes of the Marrakech area (Hassan I and Lalla Takerkoust).

The experimental approach used was based on the incubation of transparent enclosures containing natural phytoplankton and enriched by various nitrogen and/or phosphorus concentrations. The incubation time varied from 8 to 15 days. The results of different enrichment experiences show that :

- nitrogen supply has no significant effect on phytoplankton growth ;

- phosphorus and/or nitrogen fertilization induce a high increase in phytoplankton population. Phosphorus appears to constitute the first limiting factor of phytoplankton development in the two studied lakes in spite of their phosphorus mean concentration and their trophic level were completely different. Nitrogen appears to be the second limiting factor of the primary production in the two lakes.

\section{Introduction}

Pour faire face aux besoins en eau de plus en plus croissants, le Maroc s'est doté d'une importante infra-

\footnotetext{
1. Université Cadi Ayyad, Faculté des sciences, Département de biologie, B.P. S 15, Marrakech, Maroc.

2. Centre d'Ecologie des Systèmes Aquatiques Continentaux, UMR C 5576 CNRS, Université Paul Sabatier, 118 Route de Narbonne, F 31062 Toulouse Cedex 4, France.
}

structure de grands barrages et de retenues collinaires. Ceci a permis de mobiliser une grande partie des eaux de surface en vue de l'irrigation, de la production hydroélectrique et de l'alimentation en eau potable.

Toutefois, la faisabilité et la gestion de ces ouvrages aménagés se trouvent confrontées à deux problèmes : l'envasement et l'eutrophisation. Ces deux phénomènes sont principalement favorisés par le climat actuellement de plus en plus sec et les caractéristiques intrinsèques des bassins versants. 
Dans la région de Marrakech, les études déjà réalisées sur le terrain (Loudiki 1990, Loudiki et al. 1991, Hasnaoui 1992, Cherifi 1992) n'ont pas permis d'établir la primauté d'un facteur donné sur le développement du phytoplancton ; une approche expérimentale, déjà testée antérieurement (Schelske \& Stoermer 1972, Schelske et al. 1974, Stoermer al. 1978, Reynolds 1986, Donk et al. 1989), a donc été utilisée : des enceintes placées in situ et enrichies en nutriments.

Le but de ce travail est de déterminer le ou les facteurs limitants de la croissance du phytoplancton dans deux lacs réservoirs (Hassan I et Lalla Takerkoust) ; à cet effet, des enceintes placées in situ ont été enrichies en azote et en phosphore.

\section{Matériel et méthodes}

\subsection{Présentation des deux lacs - réservoirs}

Deux lacs réservoirs au nord de Marrakech ont été choisis, le lac Lalla Takerkoust, établi sur l'oued N'Fis affluent rive gauche de l'oued Tensift, et le lac Hassan I établi sur l'oued Lakhdar affluent rive gauche de l'oued Oum Er Rbia (Fig.1).

Ces deux réservoirs ont été choisis d'une part parce qu'ils se différencient par leur niveau trophique, leur régime thermique et leurs caractéristiques morphométriques et d'autre part parce que les principaux aspects de leur fonctionnement sont déjà connus (Loudiki 1990, Harrak 1991 et Cherifi 1992).

Le lac Lalla Takerkoust, mis en eau dès les années 30 , est eutrophe à hypereutrophe, peu profond et sans stratification thermique stable. A l'inverse, le lac Hassan I, de mise en eau récente (1986), est oligotrophe, très profond avec une.stratification thermique stable et régulière. Le tableau I résume les principales caractéristiques morphométriques et hydrologiques des deux retenues.

Les expérimentations in situ ont été menées en 1990 dans le lac Hassan I et en 1991 et 1992 dans le lac Lalla Takerkoust, au niveau du secteur le plus profond. Les points d'échantillonnage, retenus également pour le suivi des autres variables limnologiques, peuvent être considérés comme représentatifs des deux lacs (Hasnaoui 1992, Cherifi 1992).

\section{2 . Dispositif expérimental}

\subsubsection{Lac Hassan I}

Trois enceintes imperméables en polyéthylène transparent $(50 \mathrm{~cm}$ de diamètre, $1.50 \mathrm{~m}$ de hauteur et une capacité de $100 \mathrm{~L}$ d'eau) sont remplies chacune par 60 litres d'eau prélevés dans la colonne 0 - $5 \mathrm{~m}$ de profondeur et préalablement filtrés sur une toile de $150 \mu \mathrm{m}$ pour éliminer le zooplancton. Ces enceintes, dont la partie supérieure demeure en contact direct avec l'atmosphère, sont placées verticalement sur un support flottant à un mètre de profondeur de telle façon que les $2 / 3$ remplis de l'enceinte se trouvent immergés dans l'eau.

Le phytoplancton se trouve ainsi isolé du reste du lac et soumis aux mêmes conditions de température et d'éclairement que celles régnant dans la couche d'eau superficielle. Deux de ces enceintes sont ensuite enri-
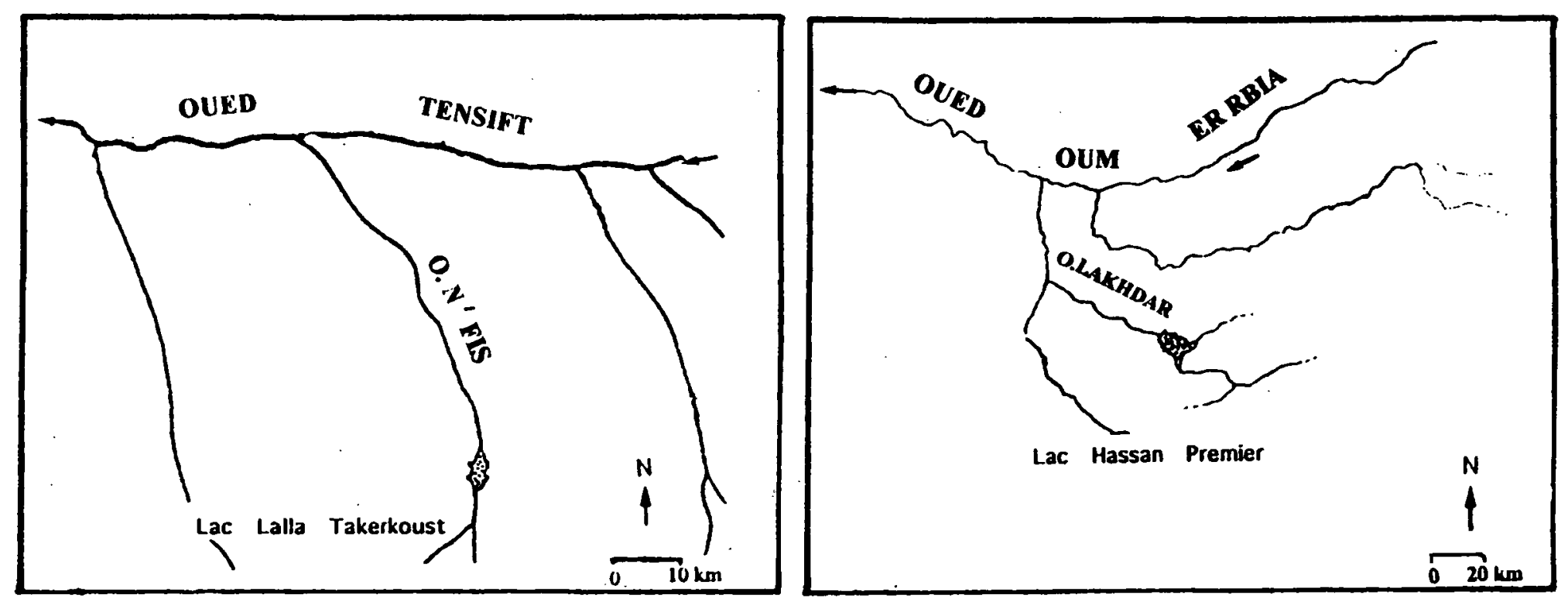

Fig. 1. Localisation géographique des deux lacs-réservoirs étudiés .

Fig. 1. Geographical location of the two studied lakes. 
Tableau 1. Caractéristiques morphométriques et hydrologiques des deux retenues de barrage Hassan I et Lalla Takerkoust.

Table 1. Morphological and hydrological characteristics of the two lakes : Hassan I and Lalla Takerkoust.

\begin{tabular}{lll}
\hline & Lac Hassan I & Lac Lalla Takerkoust \\
\hline Mise en eau & 1986 & 1935 \\
Altitude & $860 \mathrm{~m}$ & $500 \mathrm{~m}$ \\
Longueur & $4,5 \mathrm{~km}$ & $7 \mathrm{~km}$ \\
Largeur maximale & $2 \mathrm{~km}$ & $1 \mathrm{~km}$ \\
Superficie au niveau normal & $670 \mathrm{ha}$ & $584 \mathrm{ha}$ \\
Profondeur maximale & $110 \mathrm{~m}$ & $30 \mathrm{~m}$ \\
Profondeur moyenne & $40 \mathrm{~m}$ & $15 \mathrm{~m}$ \\
Volume total d'eau à la côte normale & $270 \mathrm{Mm}^{3}$ & $48,6 \mathrm{Mm}^{3}$ \\
Volume aux plus hautes eaux & $300 \mathrm{Mm}^{3}$ & $75 \mathrm{Mm}^{3}$ \\
Débit moyen annuel entrant & $10,4 \mathrm{~m}^{3} / \mathrm{s}$ & $7,59 \mathrm{~m}^{3} / \mathrm{s}$ \\
Temps moyen de renouvellement & $416 \mathrm{jours}$ & 74 jours \\
Taux d'envasement moyen & $0,8 \mathrm{Mm} 3 /$ an & $0,5 \mathrm{Mm}^{3} /$ an \\
Barrage & en terre & en béton \\
Stratification thermique & stable & sans \\
Régime thermique & Monomictique chaud & Monomictique chaud \\
Degré de trophie & Oligotrophe & Eutrophe \\
\hline
\end{tabular}

chies, l'une en azote (Enceinte EN), l'autre en phosphore (Enceinte EP). La troisième enceinte est utilisée comme témoin (ET).

Deux séries d'expériences d'enrichissement ont été réalisées durant l'hiver (du 21.02. au 08.03.1990) et le printemps (du 23.03. au 05.04.1990).

L'utilisation de grandes enceintes au niveau du lac Hassan I présente l'avantage d'expérimenter avec un grand volume d'eau et le phytoplancton naturel. Toütefois, la manipulation de ces grandes enceintes présente beaucoup de difficultés liées à leur installation et surtout à leur maintien pendant une durée suffisante dans le lac. En effet, plusieurs séries d'expériences d'enrichissement n'ont pu aboutir (automne 1989, début de l'hiver et été 1990) à cause des vents fréquents provoquant de fortes agitations et des apport d'eau dans les enceintes . C'est pour cela que dans le lac Lalla Takerkoust, nous avons opté pour l'utilisation d'un dispositif expérimental basé sur des enceintes de faible capacité.

\subsubsection{Lac Lalla Takerkoust}

Plusieurs enceintes imperméables en polyéthylène transparent (largeur $22 \mathrm{~cm}$ et longueur $36,5 \mathrm{~cm}$ ) sont remplies chacune d'un volume de 1 litre d'eau prélevée à $1.5 \mathrm{~m}$ de profondeur et préalablement filtrée sur une toile de $150 \mu \mathrm{m}$ pour éliminer une grande partie du zooplancton. Chaque enceinte contenant le phytoplancton du lac est enrichie, soit en azote $\left(\mathrm{KNO}_{3}\right)$, soit en phosphore $\left(\mathrm{K}_{2} \mathrm{HPO}_{4}\right)$, soit en azote + phosphore $\left(\mathrm{KNO}_{3}\right.$ plus $\left.\mathrm{K}_{2} \mathrm{HPO}_{4}\right)$, selon les concentrations indiquées dans le tableau 2 (A et $\mathrm{B}$ ). L'ensemble des en- ceintes enrichies est incubé in situ à la profondeur de $1.5 \mathrm{~m}$ sous des conditions de température et de lumière supposées être optimales. En effet, d'après les profils verticaux de production primaire, la profondeur de $1.5 \mathrm{~m}$ présente le maximum d'activité photosynthétique (Cherifi 1992).

Deux séries d'expériences d'enrichissement ont été réalisées, l'une au printemps (du 27.05. au 05.06.92) et l'autre en été (du 02.07.92 au 14.07.92).

\subsection{Analyse qualitative et quantitative du phyto- plancton}

Au début et à la fin de chaque expérience, des échantillons d'algues sont prélevés dans les enceintes et l'eau libre du lac.

Une fraction de ces échantillons destinée à l'identification et au comptage des algues est immédiatement fixé avec du formol neutre à $5 \%$ et l'autre fraction est conservée à l'état vivant afin de faciliter la détermination des algues flagellées.

Le dénombrement des cellules algales a été réalisé à l'aide d'un microscope inversé (Diavert, Leitz). Après homogénéisation de l'échantillon fixé, une fraction est versée dans une chambre de sédimentation de 5,10 , ou $25 \mathrm{ml}$, selon la densité du plancton, puis laissée à décanter durant 24 heures au moins (Utermohl 1958). Le dosage des pigments chlorophylliens a été fait par extraction dans l'acétone à $90 \%$. Les concentrations en chlorophylle a ont été calculées selon la formule donnée par Talling et Driver (1963) et les résultats sont exprimés en $\mu \mathrm{g} / \mathrm{L}$. 
Tableau 2. Concentrations en azote, en phosphore et les combinaisons en azote et en phosphore utilisées au cours des deux expériences d'enrichissements au niveau du lac de barrage Lalla Takerkoust.

Table 2 . Nitrogen and / or phosphorus concentrations used in the enrichment experiences in Lalla Takerkoust lake.

A : Expérience $\mathrm{n}^{\circ} 1:$ du 27/05/92 au 05/06/92 (10 jours d'incubation).

\begin{tabular}{ll}
\hline Enceintes & Concentrations des éléments enrichissants \\
E1 & $100 \mu \mathrm{g} / \mathrm{L}$ de phosphore \\
E2 & $1500 \mu \mathrm{g} / \mathrm{L}$ d'azote \\
E3 & $100 \mu \mathrm{g} / \mathrm{L}$ de $\mathrm{P}+1500 \mu \mathrm{g} / \mathrm{L} \mathrm{de} \mathrm{N}$ \\
E4 & $200 \mu \mathrm{g} / \mathrm{L} \mathrm{de} \mathrm{P}+1000 \mu \mathrm{g} / \mathrm{L} \mathrm{de} \mathrm{N}$ \\
\hline
\end{tabular}

\begin{tabular}{|c|c|}
\hline Enceintes & Concentrations des éléments enrichissants \\
\hline E1 & $50 \mu \mathrm{g} / \mathrm{L}$ de phosphore \\
\hline $\mathrm{E} 2$ & $100 \mu \mathrm{g} / \mathrm{L}$ de $P$ \\
\hline E3 & $200 \mu \mathrm{g} / \mathrm{L}$ de $P$ \\
\hline E4 & $100 \mu \mathrm{g} / \mathrm{L}$ d'azote \\
\hline E5 & $1000 \mu \mathrm{g} / \mathrm{L}$ de $\mathrm{N}$ \\
\hline E6 & $1500 \mu \mathrm{g} / \mathrm{L}$ de $\mathrm{N}$ \\
\hline E7 & $50 \mu \mathrm{g} / \mathrm{L}$ de $P+100 \mu \mathrm{g} / \mathrm{L}$ de $N$ \\
\hline $\mathrm{E} 8$ & $50 \mu \mathrm{g} / \mathrm{L}$ de $\mathrm{P}+1000 \mu \mathrm{g} / \mathrm{L}$ de $\mathrm{N}$ \\
\hline E9 & $50 \mu \mathrm{g} / \mathrm{L}$ de $\mathrm{P}+1500 \mu \mathrm{g} / \mathrm{L}$ de $\mathrm{N}$ \\
\hline E10 & $100 \mu \mathrm{g} / \mathrm{L}$ de $\mathrm{P}+1000 \mu \mathrm{g} / \mathrm{L}$ de $\mathrm{N}$ \\
\hline E11 & $100 \mu \mathrm{g} / \mathrm{L}$ de $\mathrm{P}+1500 \mu \mathrm{g} / \mathrm{L}$ de $\mathrm{N}$ \\
\hline E12 & $200 \mu \mathrm{g} / \mathrm{L}$ de $P+100 \mu \mathrm{g} / \mathrm{L}$ de $\mathrm{N}$ \\
\hline E13 & $200 \mu \mathrm{g} / \mathrm{L}$ de $\mathrm{P}+1000 \mu \mathrm{g} / \mathrm{L}$ de $\mathrm{N}$ \\
\hline E14 & $200 \mu \mathrm{g} / \mathrm{L}$ de $P+1500 \mu \mathrm{g} / \mathrm{L}$ de $N$ \\
\hline
\end{tabular}

\section{Résultats}

\subsection{Le lac Hassan I}

L'enrichissement des enceintes est de $+1 \mathrm{mg}$ de Na$\mathrm{NO}_{3} / \mathrm{L}$ pour l'enceinte $\mathrm{EN}$ et $\mathrm{de}+0.05 \mathrm{mg} \mathrm{de}$ $\mathrm{K}_{2} \mathrm{HPO}_{4} / \mathrm{L}$ pour l'enceinte EP.

\subsubsection{Expérience 1 (du 21.02. au 08.03.90)}

L'eau du lac isolée dans les enceintes au début de l'expérience se caractérise par un phytoplancton codominé par 3 groupes : les Diatomophycées (39\%), les Dinophycées (35\%) et les Chlorophycées (25\%). Cyclotella ocellata Pant. domine le groupe des Diatomées avec un maximum de $20.10^{4}$ cell../L. Les Peridiniens sont représentés par deux espèces : Peridinium cinctum (O.F .Muller) Ehr. (7.104 cell./L) et Peridiniopsis elpatiewskyi (Ostenf.) Bourr. (14.104 cell./L). Les Chlorophycées sont représentés par plusieurs espèces de Chlorococcales (Chodatella ciliata (Lagerh.) Lem, Elakatothrix gelatinosa Wille, Scenedesmus ac- cuminatus (Lagerh.) Chod. (A.Br.) Hansg, Sc. ecornis (Lem) Arlstron et Tetraedron minimum Hansg. et de Desmidiales (Cosmarium laeve (Gay) Nordst. et Cosmarium subprotumidum Nordst.).

Après 15 jours d'incubation, l'enceinte témoin (ET) présente une légère augmentation de la densité algale (Fig. 2A) (105.104 cell./L au lieu de $60.10^{4}$ cell./L au début de l'expérience). La composition du peuplement algal reste cependant similaire à celle du lac au début de l'expérience (Fig. 2B).

A l'inverse, l'enceinte enrichie en phosphore (EP) présente un développement important du phytoplancton (soit $22.10^{6}$ cell./L) en fin d'incubation (Fig. 2A). Cette forte croissance phytoplanctonique débute dès le

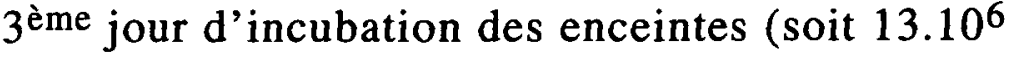
cell./L).

Parallèlement à cette forte croissance, on note la prolifération de certaines espèces très faiblement repré- 

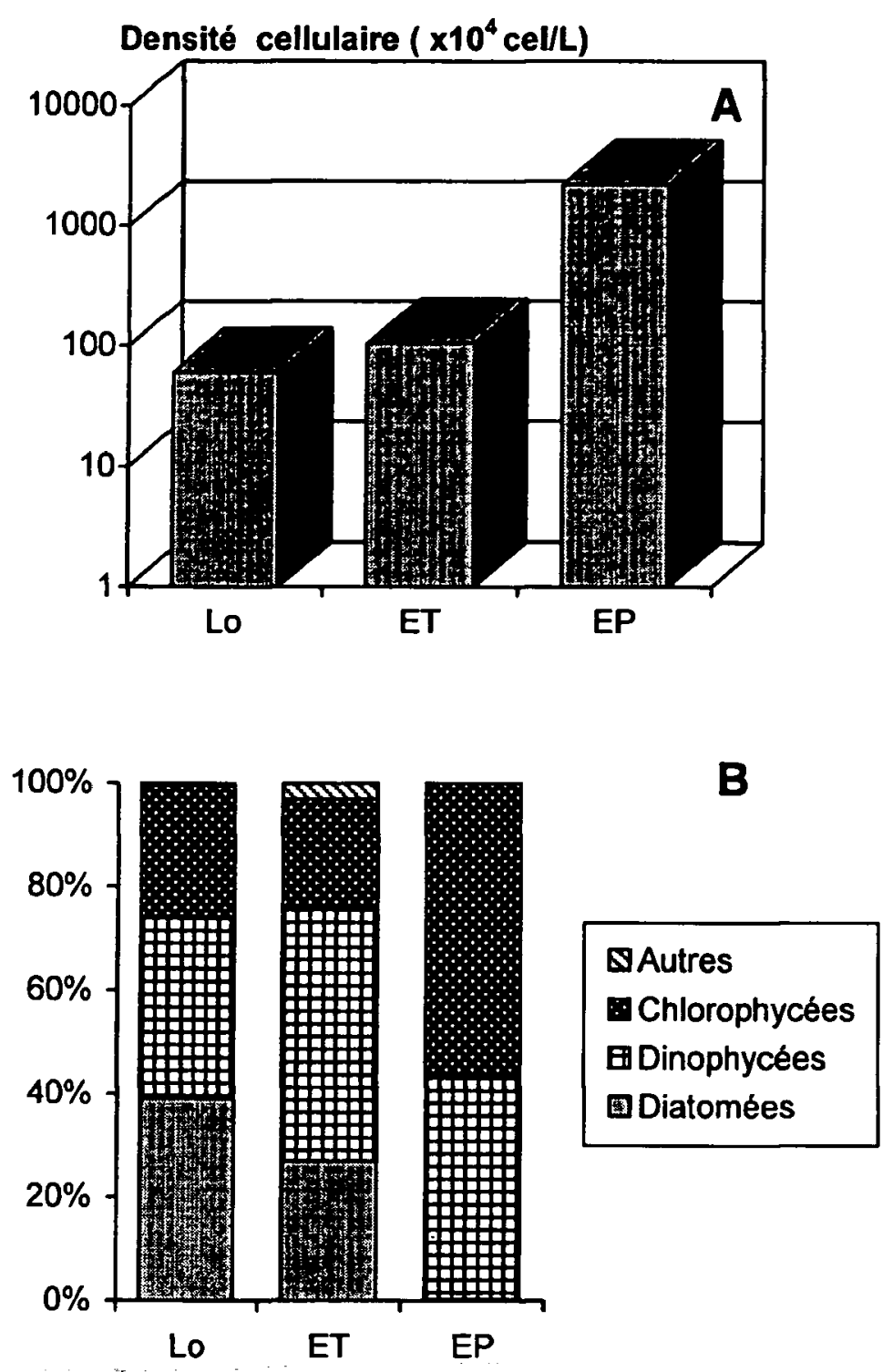

Fig. 2. Evolution de la densité cellulaire (A) et des abondances relatives des principaux groupes d'algues (B) dans l'eau libre du lac (Lo) et à l'intérieur des enceintes témoin (ET) et enrichie en phosphore (EP). Lac Hassan I.

Fig. 2. Development of cellular density (A) and relative abundance of major algae (B) in the lake water (Lo) and control (ET) and phosphorus enriched enclosures (EP). Lake Hassan I.

sentées dans l'enceinte témoin. En effet, l'enrichissement en phosphore n'entraîne pas seulement une forte croissance algale mais favorise aussi le développement de certaines espèces d'algues au profit d'autres, ceci entraîne un changement de la composition du peuplement algal dans l'enceinte EP avec principalement une codominance des Chlorophycées (56\%) et des Dinophycées ( $43 \%$ ).

Parmi 12 espèces de Chlorophycées recensées dans l'enceinte EP, 3 espèces de Chlorococcales réagissent favorablement à l'apport de phosphore : Elakatothrix gelatinosa Wille, Tetraedron minimum et Closteriopsis sp.

Les Dinophycées sont largement dominés par Peridiniopsis elpatiewskyi (soit $90 \%$ ). En effet, la période d'expérimentation a coïncidé avec le début de la phase de croissance de cette espèce qui se trouve alors fortement stimulée par l'ajout du phosphore. Ce résultat corrobore les observations faites par Gayral (1954), Derraz (1987) et Loudiki (1990) qui présentent Peridiniopsis elpatiewskyi comme caractéristique des eaux à tendance eutrophe.

A l'inverse, les Diatomées relativement dominantes au début de l'incubation, déclinent nettement après ajout du phosphore. Ceci peut s'expliquer par les faibles teneurs en silice $\left(<1 \mathrm{mg} / \mathrm{L} \mathrm{de} \mathrm{SiO}_{2}\right)$ et par la faible compétitivité des Diatomées vis-à-vis du phosphore. En effet, un faible rapport Si/P favoriserait plutôt la croissance des Chlorophycées au profit de celle des Diatomées (Tilman \& Kiesling 1984, Kilham 1986).

\subsubsection{Expérience 2 (du 23.03 au 05.04.90)}

$\left(\mathrm{EN}=+1 \mathrm{mg}\right.$ de $\mathrm{NaNO}_{3} / \mathrm{L}$ et $\mathrm{EP}=+0.05 \mathrm{mg}$ de $\left.\mathrm{K}_{2} \mathrm{HPO}_{4} / \mathrm{L}\right)$.

$\mathrm{Au}$ début de l'incubation, la composition du phytoplancton originaire du lac est similaire aussi bien dans l'enceinte témoin (ET) que dans les enceintes enrichies (EN et EP). Le peuplement algal est essentiellement dominé par les Chlorophycées (42 à $57 \%$ ) et les Dinophycées (39 à $47 \%$ ). Après deux semaines d'incubation, l'ajout de l'azote (Fig. 3) ne semble influencer ni la composition ni la croissance du phytoplancton contrairement à l'ajout du phosphore qui entraîne non seulement une forte croissance de la densité algale, mais aussi un changement de la composition du phytoplancton.

Ce sont surtout les Chlorophycées qui réagissent favorablement à l'enrichissement en phosphore et dominent largement le phytoplancton total (soit $90 \%$ ). Parmi les 8 espèces de Chlorococcales recensées dans l'enceinte EP, Oocystis lacustris Chod. présente la plus forte densité (soit $20.10^{6}$ cell./L) . Cette densité dépasse largement celle obtenue par Loudiki (1990) dans l'eau libre du lac (soit $60.10^{5}$ cell./L) au mois d'août 1988. Bien que la température de l'eau dans les enceintes soit encore plus faible $\left(18^{\circ} \mathrm{C}\right)$ par rapport à l'optimum thermique d' O.lacustris (autour de $25^{\circ} \mathrm{C}$ ) (Gayral 1954), ce résultat met en évidence que l'ajout du phosphore stimule la croissance de cette chlorophycée. 

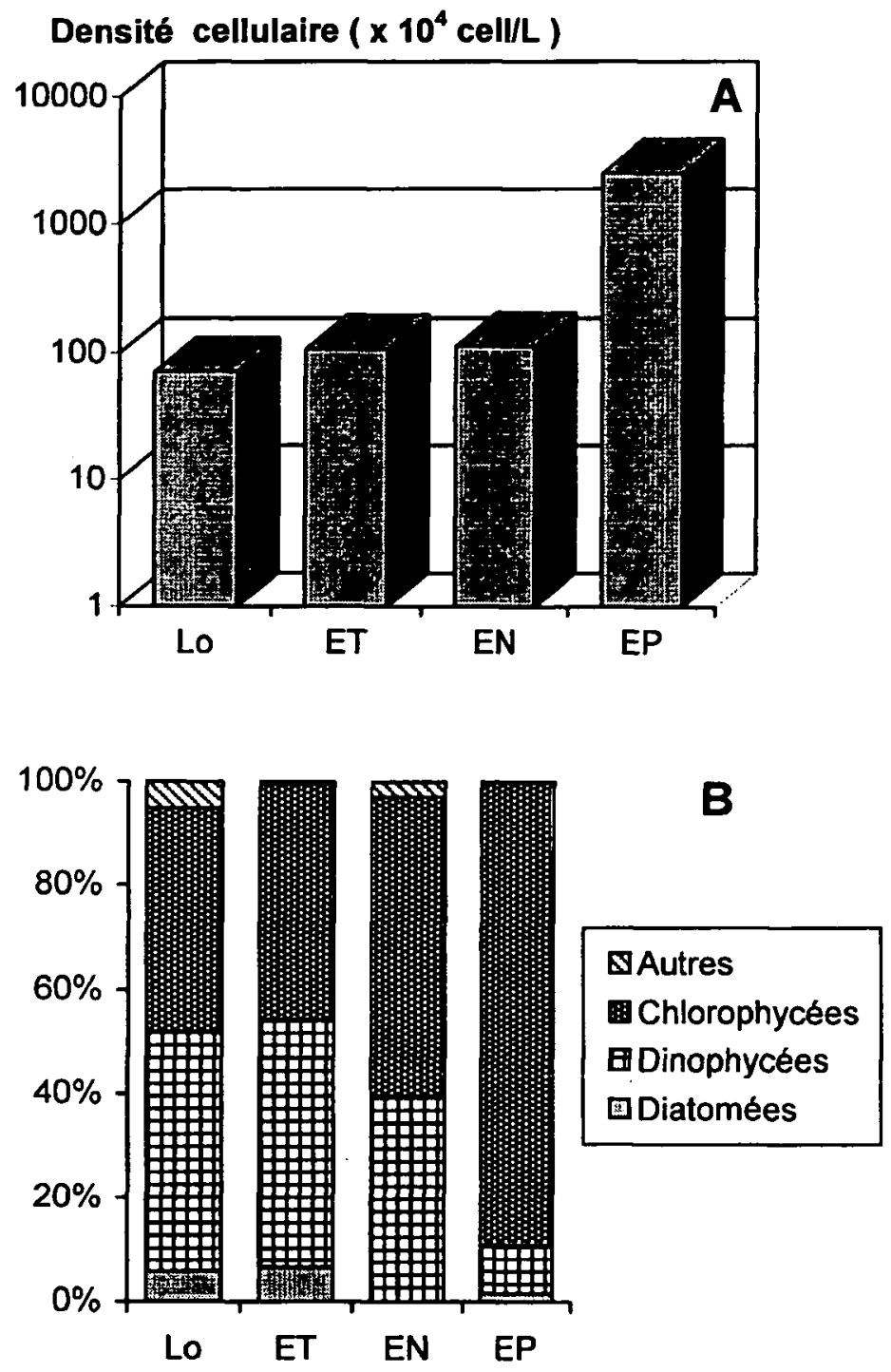

Fig. 3. Evolution de la densité cellulaire (A) et des abondances relatives des principaux groupes d'algues (B) dans l'eau libre du lac (Lo) et à l'intérieur des enceintes témoin (ET) et enrichie en azote (EN) ou en phosphore (EP). Lac Hassan I.

Fig. 3. Development of cellular density (A) and relative abundance of major algae (B) in the lake water (Lo) and control (ET) and nitogen (EN) and phosphorus enriched enclosures (EP). Lake Hassan I.

\subsection{Le lac Lalla Takerkoust}

\subsubsection{Expérience 1 ( du 27.05. au 05.06.92)}

Lo : eau du lac au début de l'incubation. L : eau du lac à la fin de l'incubation .ET : enceinte témoin. E1 : enceinte enrichie en phosphore ( $100 \mu \mathrm{g} . \mathrm{P} / \mathrm{L}), \mathrm{E} 2$ : enceinte enrichie en azote $(1500 \mu \mathrm{g} . \mathrm{N} / \mathrm{L}), \mathrm{E} 3$ : enceinte enrichie en azote $(1500 \mu \mathrm{g} . \mathrm{N} / \mathrm{L})$ et en phosphore $(100$ $\mu \mathrm{g} . \mathrm{P} / \mathrm{L}), \mathrm{E} 4$ : enceinte enrichie en azote $(1000 \mu \mathrm{g} . \mathrm{N} / \mathrm{L})$ et en phosphore $(200 \mu \mathrm{g} . \mathrm{P} / \mathrm{L})$.

L'ajout de l'azote (Fig. 4) n'entraîne aucune variation notable des teneurs en Chlorophylle a ; par contre, celles-ci montrent une forte augmentation après ajout de phosphore ou d'azote plus phosphore, avec des valeurs largement supérieures à la moyenne annuelle du lac en chlorophylle a (8 $\mu \mathrm{g} / \mathrm{L}$ Cherifi, 1992). En comparaison avec les teneurs maximales $(30 \mu \mathrm{g} / \mathrm{L}$ mesurées en novembre 1991 dans le lac (Cherifi 1992), l'ajout combiné d'azote plus phosphore stimule plus la synthèse de la chlorophylle et permet l'obtention de teneurs plus elevées (Fig.4). Cet ajout se traduit également par un fort accroissement de la densité cellulaire (maximum de $16.10^{6}$ cell./L, Fig. 4), qui reste largement supérieur à celle obtenu en avril 1991 (maximum de $5.10^{6}$ cell./L, Cherifi 1992). Cette augmentation quantitative s'accompagne d'un élargissement de la richesse spécifique ; ainsi le nombre d'espèces recensées dans l'enceinte témoin et en eau libre (8 espèces) double dans les enceintes enrichies en phosphore et en azote plus phosphore (16 espèces).

La composition du peuplement phytoplanctonique subit également des changements suite aux enrichissements en azote seul (E2) et en azote plus phosphore (E4) (Fig. 5). L'ajout de l'azote sous forme $\mathrm{KNO}_{3}$ semble favoriser la croissance de la diatomée centrique Cyclotella ocellata qui domine largement le peuplement algal (soit $90 \%$ ). Cette constatation corrobore celle faite par Le Cohu (1976) qui a montré que l'enrichissement en nitrate de potassium stimule la croissan-
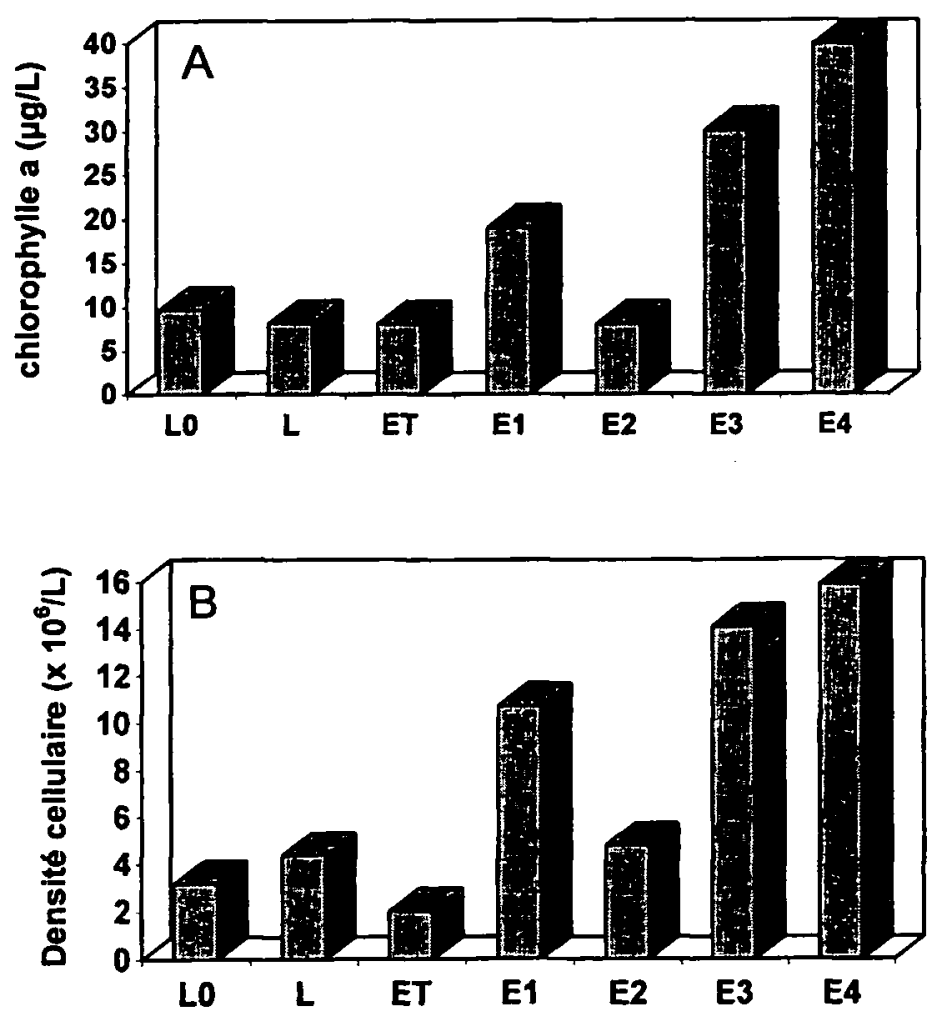

Fig. 4. Evolution de la chlorophylle a (A) et de la densité cellulaire (B) à l'intérieur des enceintes et dans l'eau libre du lac Lalla Takerkoust. Expérience 1.

Fig. 4. Development of chlorophyll a (A) and cellullar density $(B)$ in enclosures and in lake water. Experiment 1. 
ce de certaines espèces de Diatomées. Par contre dans l'enceinte enrichie en azote plus phosphore, ce sont les Chlorophycées qui dominent ( $75 \%$ ) avec un maximum de densité de $12,6.10^{6}$ cell./L pour Scenedesmus ecornis .

\subsubsection{Expérience 2 (du 02.07 au 14.07.92)}

Les enceintes E1, E2 et E3 sont enrichies en phosphore avec respectivement 50,100 et $200 \mu \mathrm{g}$. P/L. Les enceintes E4, E5 et E6 sont enrichies en azote avec respectivement 100,1000 et $1500 \mu \mathrm{g}$. N/L. Les enceintes E7 à E14 sont enrichies en azote (respectivement 100, $1000,1500,1000,1500,100,1000$ et $1500 \mu \mathrm{g}$. N/L) et en phosphore (respectivement 50, 50, 50, 100, 100, 200,200 et $200 \mu \mathrm{g}$. P/L).

Au début de l'expérience le peuplement algal est dominé par les Diatomées $(74 \%)$ et à moindre degré par les Xanthophycées (Tribonemum angustissimum $\mathrm{Pa}$ - scher) $(12,8 \%)$ et les Chlorophycées $(11,6 \%)$. On retrouve cette même composition dans l'enceinte témoin et en eau libre à la fin de l'expérience (Fig. 6). L'ajout de l'azote seul n'entraîne aucun effet sur la croissance des algues, cependant l'ajout du phosphore seul (E2) et du phosphore plus l'azote (E7 à E14) entraîne une forte augmentation de la densité du phytoplancton avec un maximum dans les enceintes enrichies en azote plus phosphore.

Des résultats tout à fait similaires ont été obtenus avec la croissance exprimée en teneurs en chlorophylle a avec un maximum de $60 \mu \mathrm{g} / \mathrm{L}$ dans les enceintes enrichies en azote plus phosphore correspondant au double des teneurs maximales mesurées dans le lac par Cherifi (1992). De même ces enrichissements entraînent un changement de la composition du phytoplancton en favorisant le développement des Chlorophycées (50 à $94 \%$ du peuplement total) au détriment de celui
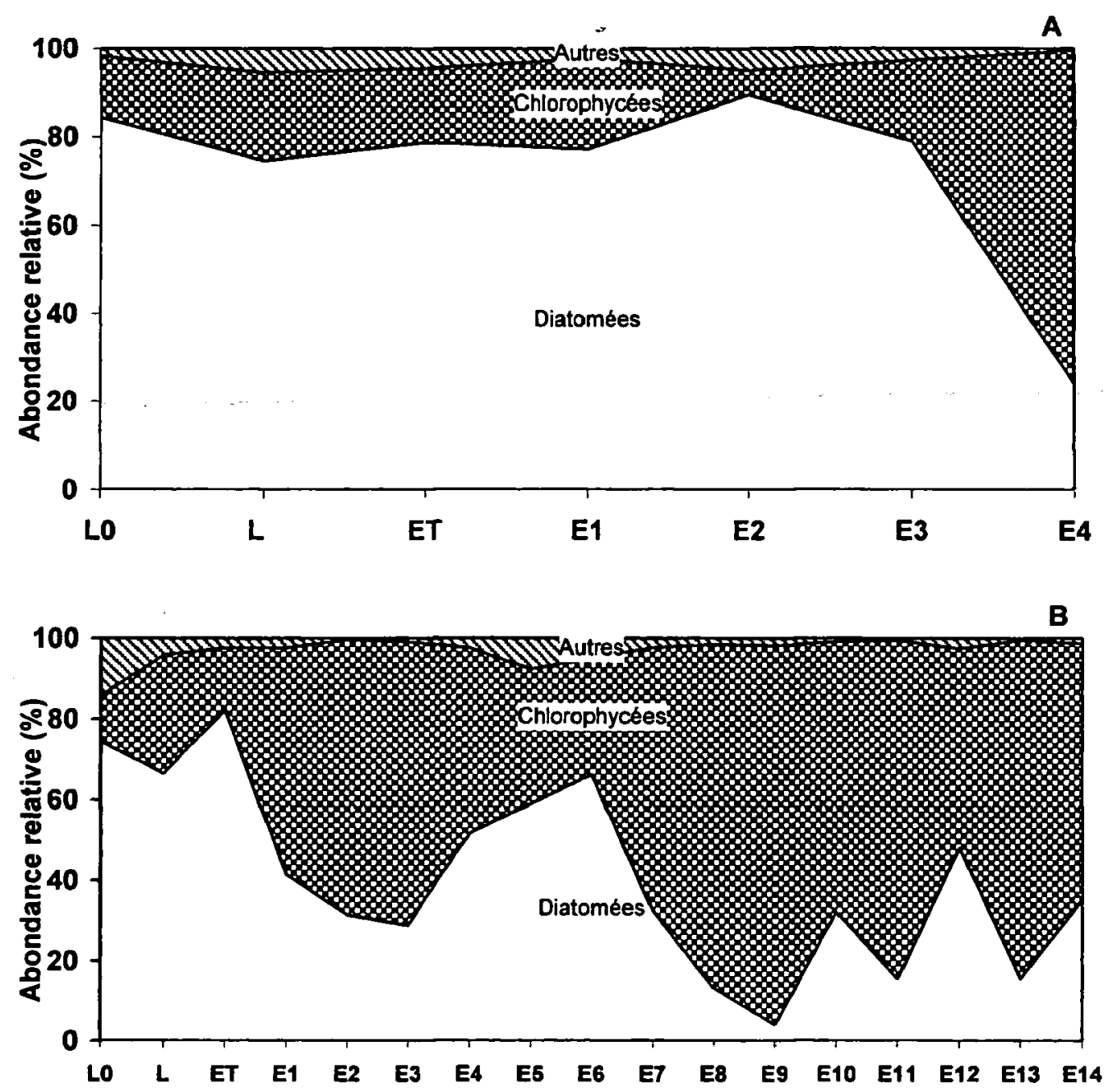

Fig. 5.A. : Evolution des abondances relatives des principaux groupes d'algues à l'intérieur des enceintes et dans l'eau libre du lac Lalla Takerkoust. Expérience 1. B : Evolution des abondances relatives des principaux groupes d'algues à l'intérieur des enceintes et dans l'eau libre du lac Lalla Takerkoust. Expérience 2.

Fig. 5.A. : Development of relative abundance of major algae in enclosures and in lake water. Experiment 1. B : Development of relative abundance of major algae in enclosures and in lake water. Experiment 2. 
des Diatomées. Cette prolifération est dûe à la croissance de 3 espèces de Chlorococcales : Oocystis lacustris, Schroederia sp et Scenedesmus ecornis.

Ces mêmes types d'enrichissements semblent agir également sur la composition spécifique des Diatomées. Dans les enceintes enrichies en phosphore seul et en azote plus phosphore c'est surtout l'espèce Nitzschia dissipata qui domine au détriment de Cyclotella ocellata. Ceci pourrait être lié au caractère benthique fréquent chez cette Diatomée Pennale dont la croissance s'est trouvée fortement stimulée à l'intérieur des enceintes enrichies en nutriments.

\section{Discussion et conclusion}

Les résultats des différentes expériences d'enrichissement en azote et en phosphore réalisées dans les deux lacs - réservoirs Hassan I et Lalla Takerkoust ont permis de dégager les points suivants :

L'ajout de l'azote seul n'a aucun effet sur la croissance algale ; les enrichissements en phosphore et en azote plus phosphore entraînent un fort accroissement du peuplement phytoplanctonique, le phosphore semble donc constituer le premier facteur limitant la croissance phytoplanctonique dans les deux lacs étudiés bien que leur concentration moyenne en phosphore et leur degré de trophie soient complètement différents. Cette limitation par le phosphore ne semble pas seulement déterminante en période chaude estivale où les teneurs en orthophosphate dans le lac sont très faibles voire nulles, mais aussi en période hivernale et printanière où ces mêmes teneurs sont souvent maximales suite aux apports du bassin versant. Ceci met également en évidence que les besoins en phosphore sont différents d'un groupe d'algues à un autre et donc d'une saison à une autre ; les espèces d'algues ayant une constante de demi-saturation (Ks) élevée pour le phosphore (essentiellement les Diatomées) prolifèrent surtout en hiver et au printemps tandis que celles à Ks faible (forte affinité pour les nutriments) (comme les chlorophycées) se développent principalement en été (Kilham 1986, Tilman et al. 1986). L'azote semble
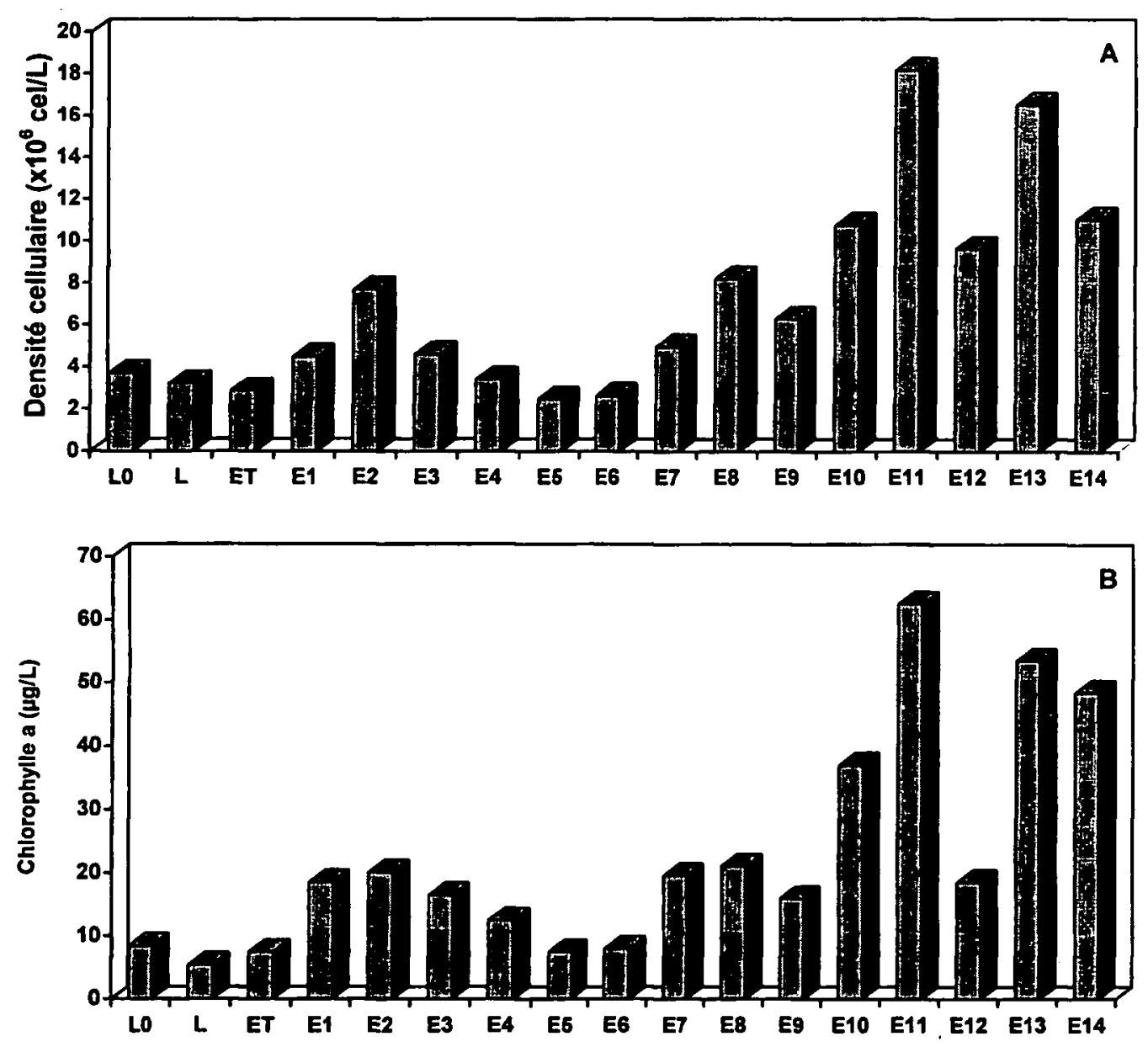

Fig. 6.A. : Evolution de la densité cellulaire à l'intérieur des enceintes et dans l'eau libre du lac Lalla Takerkoust. Expérience 2. B : Evolution des teneurs en chlorophylle a à l'intérieur des enceintes et dans l'eau libre du lac Lalla Takerkoust. Expérience 2.

Fig. 6.A : Development of cellular density in enclosures and in lake water. Experiment 2. B : Development of chlorophyll $\mathrm{a}$ in enclosures and in lake water. Experiment 2. 
constituer le second facteur limitant la production primaire dans les deux réservoirs. Ceci n'est pas étonnant si on tient compte de leurs teneurs en azote relativement élevées, même en été (moyennes > $500 \mu \mathrm{g} . / \mathrm{L}$ : Loudiki 1990, Cherifi 1992). Autrement dit un premier apport important en phosphore au lac serait d'abord à l'origine de l'installation d'un état d'eutrophie. Si par la suite un apport d'azote survient, il peut aggraver la situation vers un état hypereutrophe avec changement radical et important de la composition du phytoplancton. Ce dernier peut évoluer vers la formation d'efflorescences caractérisées par la pullulation d'un seul groupe voire même d'une seule espèce. Ceci est d'ailleurs actuellement le cas dans̀ le lac Lalla Takerkoust où prolifère régulièrement en été et au début de l'automne la Cyanobactérie Microcystis aeruginosa Kütz. entraînant la formation d'une fleur d'eau spectaculaire (Sbiyyaa 1997). Il est bien évident que les conséquences de cette population de Cyanobactéries sur les différentes utilisations de l'eau du lac (eau de boisson pour la population riveraine et pour le bétail) peuvent être très néfastes.

A côté de la stimulation de la croissance, les enrichissements en phosphore et en azote plus phosphore entraînent également un changement de la composition globale du phytoplancton avec le plus souvent une large dominance des Chlorophycées (Elakatothrix gelatinosa, Tetraedron minimum, Closteriopsis sp., Oocystis lacustris, Scenedesmus ecornis, Schroederia sp.) au détriment des autres groupes en présence. Ceci va à l'encontre des observations de certains auteurs (Fraser $\&$ Duthie 1987, Stockner et al. 1980) qui n'ont obtenu aucun changement de la composition du phytoplancton suite à l'enrichissement par le phosphore. Toutefois, les travaux de Schindler \& Fee (1975) ont mis en évidence, suite à l'ajout du phosphore, un changement radical de la composition du phytoplancton avec passage de la codominance des Diatomées et des Chlorophycées à un peuplement algal représenté uniquement par les Cyanobactéries. De leur coté, Stoermer et al. (1978) ont démontré que l'ajout du phosphore favorise principalement le développement des espèces de Diatomées.

Par ailleurs, la réponse aux différents ajouts des nutriments semble dépendre largement du potentiel algal d'espèces existant initialement dans le lac. Ainsi l'enrichissement en phosphore par exemple peut stimuler la croissance des espèces présentes dans le lac et qui se trouvaient limitées par le phosphore. Les fortes densités des espèces stimulées peuvent entraîner le passage progressif des communautés d'algues limitées en phosphore à des communautés d'algues limitées en azote ou en silice. De telles variations de la disponibilité des ressources nutritives peuvent agir sur la sélection des espèces eutrophes (Reynolds 1984).

D'autre part, les expériences d'enrichissement en nutriments soulignent l'importance du rapport N/P dans le comportement des différents groupes d'algues et l'écophysiologie des espèces elles mêmes. Les réponses de ces dernières sont principalement déterminées par les variations du rapport N/P. Ceci signifie que pour un même rapport N/P la croissance peut être limitée soit par $N$ pour certaines espèces soit par $\mathrm{P}$ pour d'autres espèces (Rhee 1978, 1982, Thompson \& Rhee 1994).

D'autres expériences étalées sur toute l'année et portant sur d'autres retenues s'avèrent nécessaires pour compléter les données actuelles sur la situation des lacs-réservoirs au Maroc.

\section{Travaux cités}

Chérifi O. 1992. - Evolution et dynamique du phytoplancton en relation avec certains paramètres biotiques et abiotiques au niveau de la retenue Lalla Takerkoust (Maroc). Thèse $3^{\text {ème }}$ cycle, Univ. Cadi Ayyad Marrakech : 155 p.

Derraz M. 1987. - Influence des facteurs environementaux sur l'évolution de la qualité des eaux de la retenue Oued Elmakhazine (Maroc). Thèse $3^{\text {ème }}$ cycle, Université Paul Sabatier, Toulouse : $95 \mathrm{p}$.

Donk E.V., Mur L.R \& Ringelberg J. 1989. - A study of phosphate limitation in lake maarsseveen : phosphate uptake kinetics versus bioassays. Hydrobiologia, 188/189 : 201-209.

Fraser P.C. \&. Duthie H.C. 1987. - Response of the phytoplankton community to weekly additions of monoammonium phosphate in a dystrophic lake. Arch. Hydrobiol.110 (1) : 67-82.

Gayral P. 1954. - Recherches phytolimnologiques au Maroc. Thèse doctorat es-sciences, Univ. Paris : 301 p.

Harrak R. 1991. - Eutrophisation du lac de barrage Lalla Takerkoust. Equilibre Nitrification - Denitrification. Thèse $3^{\text {ème }}$ cycle, Univ. Cadi Ayyad, Marrakech : 159 p.

Hasnaoui M. 1992. - Biomasse, production primaire et distributions horizontale et verticale du phytoplancton dans le lac - réservoir Hassan $1^{\text {er }}$ (Maroc). Thèse $3^{\text {emme }}$ cycle, Univ. Cadi Ayyad., Marrakech : $135 \mathrm{p}$.

Kilham S.S. 1986. - Dynamics of lake Michigan natural phytoplankton communities in continuous cultures along $\mathrm{Si}$ : P loading gradient. Can J. Fish. Aquat. Sci., 43 : 351-360.

Le Cohu R. 1976. - Effet de l'enrichissement continu en nitrates sur les populations de Diatomées isolées à l'aide d'enceintes expérimentales placées dans un étang oligotrophe. Int. Revue ges. Hydrobiol., 61 (6): 789-798.

Loudiki M. 1990. - Etude limnologique d'un hydrosystème récemment aménagé dans la région de Marrakech (Maroc). Apports du bassin versant, Hydrochimie, Algues et Macrophytes. Thèse de Doctorat es-Sciences, Univ. Aix-Marseille III : 353 p.

Loudiki M., Bouterfas R. \& Mouhri K. 1991. - Dynamique et évaluation des apports du bassin versant et de l'état trophique du lac réservoir Hassan I. Maroc. Rev. Fac. Sci. Mar., n ${ }^{\circ} 8: 45-47$.

Rhee G.Y. 1978. - Effects of N/P atomic ratios and nitrate limitation on algal growth, cell composition, and nitrate uptake. Limnol. Oceanogr., $23: 10-24$. 
Rhee G.Y. 1982. - Effects of environmental factors and their interactions on phytoplankton growth. Adv. Microbiol. Ecol., 6 : 33-74.

Reynolds C.S. 1984. - The ecology of freshwater phytoplankton. Cambridge University Press, Cambridge : $384 \mathrm{p}$.

Reynolds C.S. 1986. - Experimental manipulations of the phytoplankton periodicity in large limnetic enclosures in Blelham Tarn, English lake District. Hyrobiologia, 138 : 43-64.

Schelske C.L., Rothman E.D., Stoermer E.F. \& Santiago M.A. 1974. - Responses of phosphorus limited lake Michigan phytoplankton to factorial enrichments with nitrogen and phosphorus. Limnol. Oceanogr., 19 : 409-419.

Schelske C.L. \& Stoermer E.F. 1972. — Phosphorus, Silica and eutrophication of lake Michigan. In : G.E. Likens (ed.), Nutrients and eutrophication. Spec. Symp. I, Am. Soc. Limnol. Oceanogr., : 151-171.

Schindler D.W. \& Fee E.J. 1974. - Experimental lakes Area : Whole lake experiments in eutrophication. J. Fish. Res. Bd., Canada, $31: 937-953$

Sbiyyaa B. 1997. - Déterminisme, écophysiologie, toxicité et implications écologiques du bloom toxique à Microcystis aerugino$s a$ (Kütz.) (Cyanobactérie) dans le lac du barrage Lalla Takerkoust (Maroc). Thèse de $3^{\text {ème }}$ cycle, Université Cadi Ayyad, Marrakech : $219 \mathrm{p}$.

Stoermer E.F., Ladewski B.G. \& Schelske C.L. 1978. - Population reponses of lake Michigan phytoplankton to nitrogen and phosphorus enrichment. Hydrobiologia, 57 (3) : 249-265.
Stockner J.G., Shortreed K.S. \& Stephens K. 1980. - The British Columbia lake Fertilization program : Limnological results from the first 2 years of nutrient enrichment. Can. Tech. Rep. Fish. Aquat. Sci. 924.

Talling J.F. \& Driver D. 1963. - Some problems in the estimation of chlorophyll a in phytoplankton. Prediction and Measurement of photosynthetic productivity : 431-434. Proocedings of the IBP/PP techni-Hawai U.S. (Ed. M.S. Doty) : 142-146. Atomic energy commission publication TID7633.

Tilman D. \& Kiesling R.L. 1984. - - Freshwater algal ecology : Taxonomic trade-offs in the temperature dependence of nutrient competitive abilities. In M.J. Klug and C.A. Reddy (ed.). Current perspectives in microbiol ecology. American Society for Microbiology, Washington DC. : 314-319.

Tilman D., Kiesling R.L., Stoermer R., Kilham S.S. \& Johnson F.A. 1986. - Green, Bluegreen and diatom algae : Taxonomic differences in competitive ability for phosphorus silicon and nitrogen. Arch. Hydrobiol., 106 : 473-485.

Thompson P.A. \& Rhee G.Y. 1994. - Phytoplankton responses to eutrophication in Algae and water pollution. Ergbenisse der Limnologie - Advances in Limnology, 42 : 125-166.

Utermöhl H. 1958. - Zur Vervollkommnung der quantitaven Phytoplankton-Methodik. Mitt. int. Ver. theor. angew. Limnol., 9 : 1-38. 